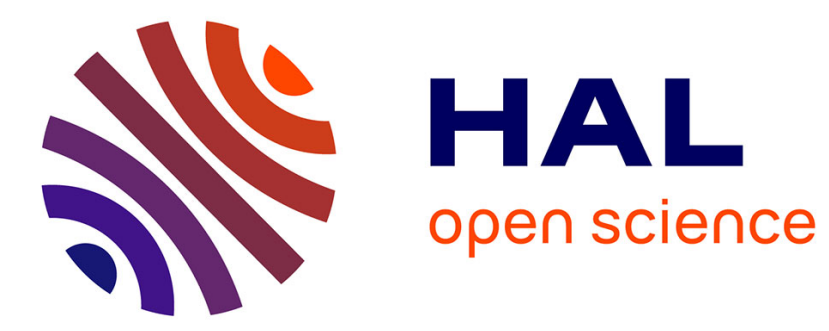

\title{
Control of cable-driven manipulators in the presence of friction
}

Salih Abdelaziz, Laurent Barbé, Pierre Renaud, Michel de Mathelin, Bernard Bayle

\section{- To cite this version:}

Salih Abdelaziz, Laurent Barbé, Pierre Renaud, Michel de Mathelin, Bernard Bayle. Control of cable-driven manipulators in the presence of friction. Mechanism and Machine Theory, 2017, 107, pp.139-147. 10.1016/j.mechmachtheory.2016.09.014 . lirmm-01878600

\section{HAL Id: lirmm-01878600 \\ https://hal-lirmm.ccsd.cnrs.fr/lirmm-01878600}

Submitted on 21 Sep 2018

HAL is a multi-disciplinary open access archive for the deposit and dissemination of scientific research documents, whether they are published or not. The documents may come from teaching and research institutions in France or abroad, or from public or private research centers.
L'archive ouverte pluridisciplinaire HAL, est destinée au dépôt et à la diffusion de documents scientifiques de niveau recherche, publiés ou non, émanant des établissements d'enseignement et de recherche français ou étrangers, des laboratoires publics ou privés. 


\title{
Control of cable-driven manipulators in the presence of friction
}

\author{
S. Abdelaziz*, L. Barbé, P. Renaud, M. de Mathelin, B. Bayle \\ Laboratoire des sciences de l'Ingénieur, de l'Informatique et de l'Imagerie, Strasbourg University, 67412 Illkirch, France
}

\section{A R T I C L E I N F O}

\section{Keywords:}

Cable-driven manipulators

Motion control

Redundant robots

\begin{abstract}
A B S T R A C T
Cable-driven systems are of great interest in applications for which remote actuation is required, like for instance to operate within medical imaging devices. In such applications, robotic architectures based on remotely actuated cable-driven manipulators (CDM) offer innovative design options. However, unlike more conventional CDM, such designs involve specific control problems. This paper deals with the control of CDM in the presence of friction, as experienced when particular design constraints have to be taken into account (size, materials, length of transmissions). In this case, the influence of friction on the manipulator motions is such that dynamic modeling, identification and control, are no longer adapted, nor even possible. As a result, for quasi-static positioning tasks, we propose a CDM position control strategy resulting from a cascade structure, with an internal cable tension control loop to handle friction. Cable tension is measured using an original instrumented compliant structure. From the control point of view, the originality of the present paper comes in particular from the development of a feasibility algorithm which allows us to limit cables tensions within a specified range. This strategy is experimentally assessed on a robot for image-guided medical procedures.
\end{abstract}

\section{Introduction}

\subsection{Context and state of the art}

Though better known for their coverage of large workspaces, CDM are also of great interest to develop robotic systems with strict design constraints. This is for instance the case of manipulators designed to operate within MRI medical imagers, as shown in Fig. 1. In such a context, CDM offer alternative solutions to more conventional robotic systems, based on ultrasonic [1-3] or pneumatic actuators [4,5]. Even though fabricated with non-ferromagnetic parts to ensure MRI-compatibility, the size of these conventional robotic systems remains problematic to access areas such as the perineum within a limited and cluttered space. In [6,7], we have presented the principle and the detailed design of a CDM dedicated to percutaneous prostate interventions. The issue of CDM position control is addressed in the present paper.

A CDM is a class of parallel robots for which the limbs are cables. These cables are tensioned between the base and the platform, whose movements are obtained by modifying the cables length. The CDM stiffness depends on the tensions in the cables, which must remain above a minimum (positive) value to ensure tightening of the cables and below a maximum value to avoid cables breakage. Similar to serial/parallel manipulators, two kinds of control schemes can be considered for CDM, namely a joint space control and an operational space control. In the joint space control, the manipulator inverse kinematics is used to convert the platform desired positions into corresponding cables length. In this control framework, Kawamura et al. [8] propose a proportional derivative (PD)

\footnotetext{
* Corresponding author.

E-mail addresses: abdelaziz@lirmm.fr (S. Abdelaziz), laurent.barbe@unistra.fr (L. Barbé), pierre.renaud@insa-strasbourg.fr (P. Renaud), demathelin@unistra.fr (M. de Mathelin), bernard.bayle@unistra.fr (B. Bayle).
} 


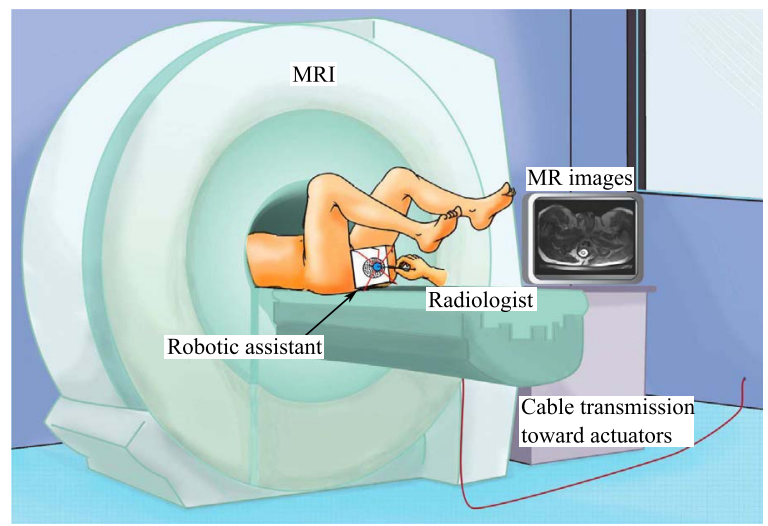

Fig. 1. MRGuide: a robotic assistant based on a CDM [7].

feedback controller for ultrahigh speed CDM. Gravity compensation as well as an internal force controller are considered so as to ensure stiffness in the system. The authors point out that the cables vibration can be reduced by increasing the internal forces. Fang et al. [9] propose a computed-torque controller in the cable length coordinates with a feedforward part of the inverse dynamics. Gouttefarde et al. [10] propose a similar computed-torque controller but with two feedforward terms, one to compensate for the platform dynamics, and the other to compensate for dry and viscous frictions of the actuators.

Regarding the control in the operational space, Gallina et al. [11] perform a trajectory tracking using a computed-torque controller. The desired acceleration is added as a feedforward term to the control law in order to improve the tracking. No experimental validation has however been reported. Kino et al. [12,13] propose to visually control the end-effector motion. To reduce the internal force errors among wires that result from the errors of the actuators location, a robust PD controller with adaptive compensation is developed. To perform this compensation, the position of the end-effector has to be measured using external sensors. To deal with the elasticity of the cables, Laroche et al. [14] propose a $H_{\infty}$ controller, derived from a linear dynamic model. No experimental validation has however been reported. Recently, Reichert et al. [15] propose a robust internal force-based impedance control using a computed-torque structure. Each actuated cable, considered as a manipulator, is controlled to have a specified impedance. The internal force, computed from the cables tension measurement via a kinematic relationship, is integrated into the impedance controller in order to reduce the effect of the platform dynamics on tracking and steady-state position errors.

\subsection{Problem and contributions}

For conventional CDM, the actuators are usually placed at the corners of the manipulator base frame and the platform moves freely in space. The cables are then directly tensioned between the actuators and the platform with a transmission using pulleys. In this configuration, friction between the actuators and the platform is relatively low if the transmission is carefully designed and fabricated. In our application, a remote actuation of the platform is considered because of MRI-compatibility constraints. Electromagnetic actuators can then be used. They are no longer placed within the manipulator base frame but at a distance using cable transmission, as shown for illustration purpose in Fig. 1. This choice has also been adopted for instance by Salimi et al. [16] to design a remotely actuated cable-driven robotic assistance for cardiac surgery under MRI. In this situation, the cables are not directly tensioned between the actuators and the platform. As a result, the control of such devices requires a particular attention, as high friction along the cable transmissions has to be handled. This issue is quite generic since it can result from many types of constraints (size, materials, length of transmissions), which may cause friction even with careful design.

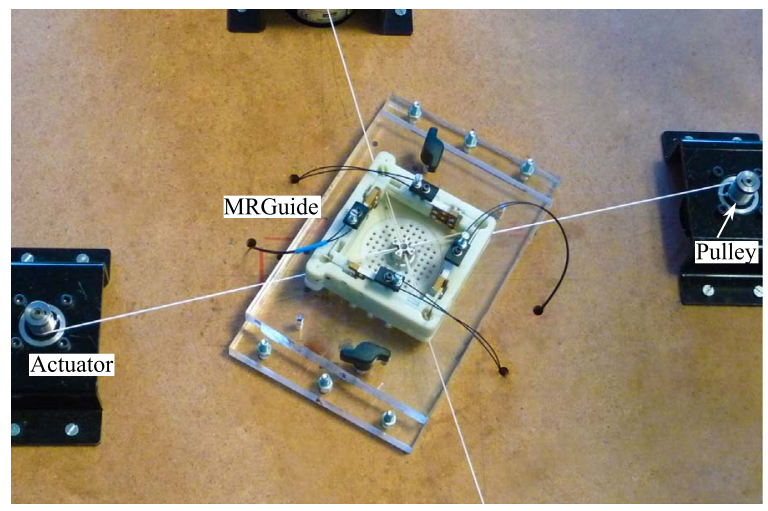

Fig. 2. Set-up for MRGuide control. 
For conventional CDM, the issues of high velocity, cables elasticity and payload manipulation are naturally of concern. These issues have led the authors to propose control schemes based on the manipulator dynamics. For the prototype device we introduced (Fig. 2), friction is the main issue. Indeed, the evaluation of friction along the line transmission [17] has shown at least 75\% tension loss for the best configuration of the cables path with the platform at the center of the workspace. In addition, this tension loss is pose-dependent. It seems therefore out of reach to explicitly model and compensate friction in the control scheme. The previous control approaches based on the manipulator dynamics or using only a position-based controller are not adapted for this class of mechanisms. Fortunately in this medical context, the trajectories to be tracked are of low velocity and the payload is negligible. It is in this framework that we propose and develop a cascade control scheme with an inner cable tension loop and an outer platform position control loop. It includes a feasibility procedure that takes into account the mechanical constraints as well as a pretension procedure that avoids the platform displacement during the cables pretension, as friction along the line transmissions is different in each cable.

The paper is organized as follows. In Section 2, an overview of the robotic assistant and a description of its instrumentation are presented. In Section 3, the cascade control structure, the feasibility and the cable pretension algorithms are introduced. An experimental evaluation of the proposed control structure and a comparison with a state of the art control approach are presented in Section 4. Conclusions and perspectives are finally developed in Section 5.

\section{System overview}

The present section aims to provide information about the system which is used to illustrate and assess the proposed control approach. For more details on the robotic assistant design, see [7]. Named MRGuide, the robot is a 2-DOFs planar CDM designed to position a needle guide with 4 cables, as shown in Fig. 3, left. The needle is in interaction with the tissues and the radiologist. These interaction forces are equal to $5 \mathrm{~N}$ in magnitude, with any direction in the base plane. The CDM must therefore be able to resist to these external forces. For this, the tensions in the cables must be admissible with values between two tension limits. The minimum tension is chosen equal to $10 \mathrm{~N}$ in order to ensure a correct tightening of the cables. The maximum tension is set to $60 \mathrm{~N}$ so as to avoid over-sizing the actuators that may cause safety issues.

An instrumentation has been developed so as to measure the cables tension at the manipulator level. Its principle relies on the use of a compliant structure that is sensitive to the tensions in the cables (Fig. 3, left). Each cable is attached to a corner of the square compliant structure (Fig. 3, left, point C) at one end, passes through the platform by making a U-turn and is winded around an actuated pulley. In order to simplify the tension evaluation, the compliant structure is designed to get a decoupling in the system, i.e. a tension $\tau_{i}$ in the cable $i$ only induces a force $f_{i}$ in the bar $i$ (Fig. 3, left). In this situation, $\boldsymbol{\tau}_{i}$ is determined from $f_{i}$ by a simple projection. To evaluate the bar deformation, an amplification mechanism (Fig. 3, right) is integrated in each bar of the compliant structure. For the bar $i$, the force compression $f_{i}$ induced by the cable tension $\tau_{i}$ is converted into a displacement of sufficient amplitude with the amplification mechanism (Fig. 3, right). The measurement of the lever tip displacements will allow to evaluate the bar deformation and therefore the cable tension $\boldsymbol{\tau}_{i}$.

For the system fabrication, Dyneema polymer cables are selected for their stiffness, their high resistance to breakage and their MRI-compatibility. The compliant structure and the frame are manufactured in rapid prototyping as a single element with glass filtered polyamide material for MRI-compatibility. The device outer dimension is about $100 \times 100 \times 40 \mathrm{~mm}^{3}$ with a weight of $120 \mathrm{~g}$. The overall system exhibits excellent compatibility with the MRI scanner, as reported in [7].
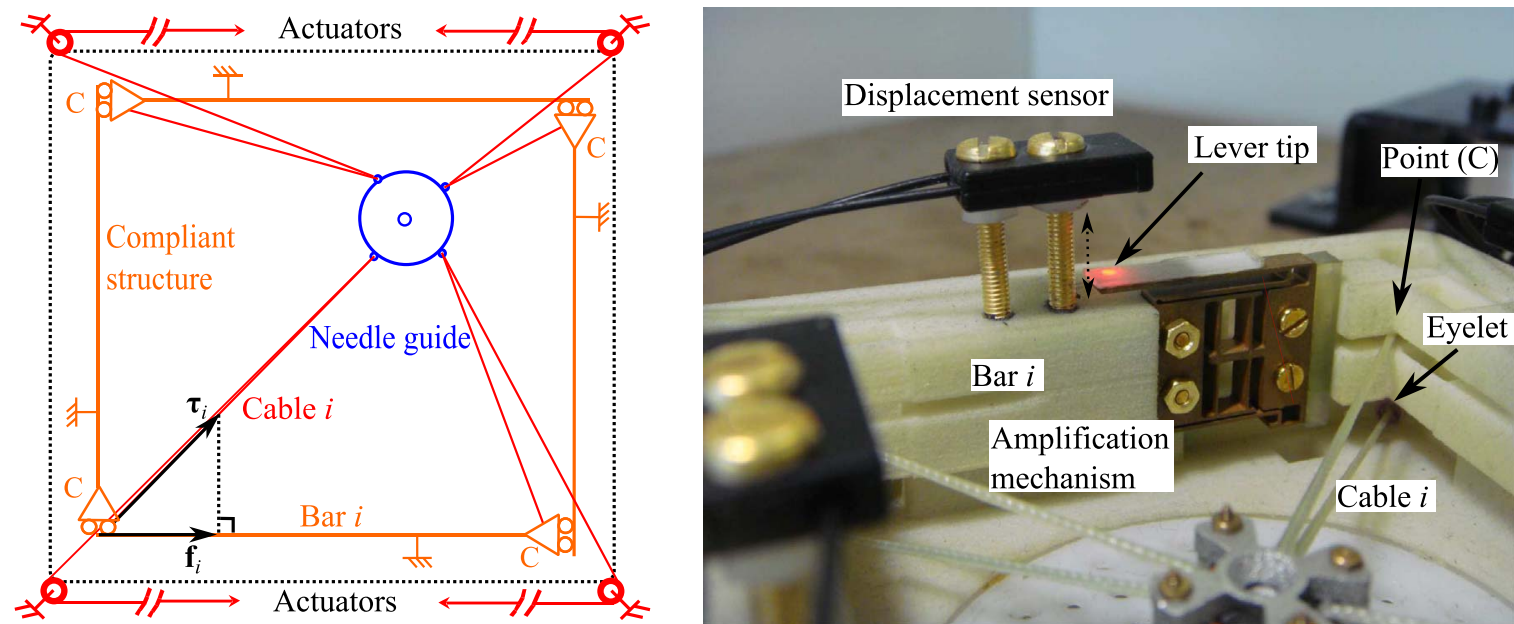

Fig. 3. Left, the path of the cables with the use of an instrumented structure. Right, cable tension evaluation using an amplification mechanism and a displacement sensor. 


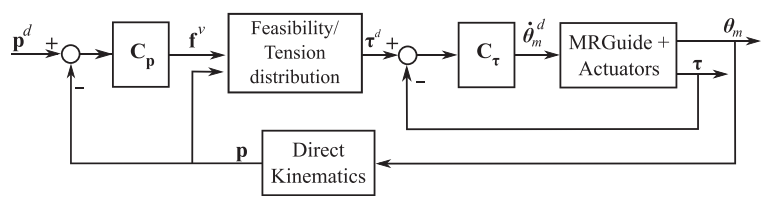

Fig. 4. Position based control with an internal loop for the tension supervision.

\section{Control}

\subsection{Position based control with tension supervision}

The proposed cascade control scheme (Fig. 4) achieves the platform position control with an inner loop controlling the cable tensions to avoid in particular tension transient peaks. To ensure stability, the inner loop must be faster than the outer loop. In the literature, the use of the tension measurements as a control feedback has been considered for conventional CDM with low friction, as proposed by [9]. This is performed in the objective of adjusting the stiffness of the manipulator by controlling the internal forces in the cables. In our case, the tensions in the cables are measured and controlled in the purpose of handling friction issues of nonconventional remote-actuated CDM. Besides, the efficiency of such a cascade strategy has been proven in other contexts, in particular for DC motor velocity control, where an internal current feedback loop is used to moderate current transient peaks. In the present paper, this CDM control strategy is developed and assessed. Note that this scheme was initially introduced in [6] and was exploited more recently by other authors [18] but for conventional CDM with low friction.

The outer loop includes a position controller $\mathbf{C}_{\mathbf{p}}$, a tension distribution algorithm and the direct kinematic model of the robot. $\boldsymbol{\theta}_{m}$ is a 4-dimensional vector that contains the angular positions of the actuators. These angular positions are used to compute, via the direct kinematic model, the platform position $\mathbf{p}=\left[\begin{array}{ll}x & y\end{array}\right]^{T}$. The platform desired position is denoted $\mathbf{p}^{d}=\left[\begin{array}{ll}x^{d} & y^{d}\end{array}\right]^{T}$. The 2-dimensional virtual force vector $\mathbf{f}^{v}$ to be applied to the platform is computed as $\mathbf{f}^{v}=\mathbf{C}_{\mathbf{p}}\left(\mathbf{p}^{d}-\mathbf{p}\right)$, where $\mathbf{C}_{\mathbf{p}}=\operatorname{diag}\left(k_{p x}, k_{p y}\right)$ is a simple proportional controller. This force is then considered as an input to the Feasibility/Tension distribution block. In this block, a feasibility algorithm verifies the feasibility to generate the virtual force $\mathbf{f}^{v}$ using admissible tensions in the cables. If this force is unfeasible, a feasible force is then computed. A tension distribution algorithm determines therefore the desired tensions in the cables $\tau^{d}$ that need to be applied to the platform. The two algorithms are explained in details hereafter. The desired tensions are then compared to the measured ones $\tau$ at the manipulator level. To cancel the disturbances due to friction along the cables transmission, a Proportional-Integral controller $\mathbf{C}_{\boldsymbol{\tau}}=\operatorname{diag}\left(c_{\boldsymbol{\tau}}, c_{\boldsymbol{\tau}}, c_{\boldsymbol{\tau}}, c_{\boldsymbol{\tau}}\right)$, is considered in the tension loop. The output of the $\mathbf{C}_{\boldsymbol{\tau}}$ controller is a 4-dimensional vector $\dot{\boldsymbol{\theta}}_{m}^{d}$ that contains the desired angular velocities of the actuators. These velocities are considered as references for the low-level control of the actuators.

The tension distribution block solves the following constrained linear system of equations:

$$
\mathbf{W} \boldsymbol{\tau}^{d}=\mathbf{f}^{v} \text { Subject to: } \quad \boldsymbol{\tau}_{\min } \leq \boldsymbol{\tau}^{d} \leq \boldsymbol{\tau}_{\max }
$$

This system of equations represents the static model. $\mathbf{W}$ is a $2 \times 4$ wrench matrix. The 4 -dimensional vectors $\boldsymbol{\tau}_{\min }$ and $\boldsymbol{\tau}_{\max }$ represent respectively the minimum and the maximum tensions in the cables. Different iterative approaches based on linear [19,9,20] or quadratic programming [21] have been considered in the literature to solve Eq. (2). The solutions obtained using linear programming are however subject to discontinuities that may induce vibrations [22]. Pott et al. [23] propose a non-iterative algebraic based approach that computes a continuous and feasible solution for cable redundancy $r=m-n=2$ with $m$ being the number of cables and $n$ the number of degree of freedom. The main drawback of this approach is its inability to find a solution to Eq. (2) in some situations, in particular when the platform is close to the workspace border while a feasible solution exists. A second noniterative approach has been developed by Mikelsons et al. [24] and has been recently improved by Gouttefarde et al. [10]. The algorithm analytically computes a feasible and a continuous solution along trajectories using the following relationship:

$$
\tau^{d}=\mathbf{W}^{+} \mathbf{f}^{v}+\mathbf{H} \lambda
$$

where $\mathbf{W}^{+}$denotes the Moore-Penrose pseudo-inverse of $\mathbf{W}, \mathbf{H}$ a basis of $\mathbf{W}$ null-space, and $\lambda$ a vector computed using a barycentrical approach [24]. Adapted for cables redundancy $r=2$ as for the MRGuide, this algorithm has been preferred and implemented in the tension distribution block of Fig. 4.

The above presented control scheme can be used only if we handle 2 issues that could cause damage during operation. The first one is feasibility assessment, to guarantee the mechanical constraints. The second one is pretension management, to avoid the platform displacement during the cables pretension phase as well as the cables tension discontinuity. These two issues are handled using two algorithms that are introduced in this paper and implemented in the Feasibility/Tension distribution block of Fig. 4.

\subsection{Feasibility and pretension algorithms}

\subsubsection{Feasibility}

The aforementioned approaches for tension distribution, including [24], are solving tools and not methods that deal with the existence of a solution. A solution to Eq. (2), expressed by the relationship (2), exists if and only if the virtual force $\mathbf{f}^{v}$ is feasible. Different iterative approaches are proposed to deal with the existence of a solution, particularity to solve the constrained differential kinematic models that are similar to the resolution of Eq. (2). An infinity-norm based approach is proposed by Deo et al. [25]. It 

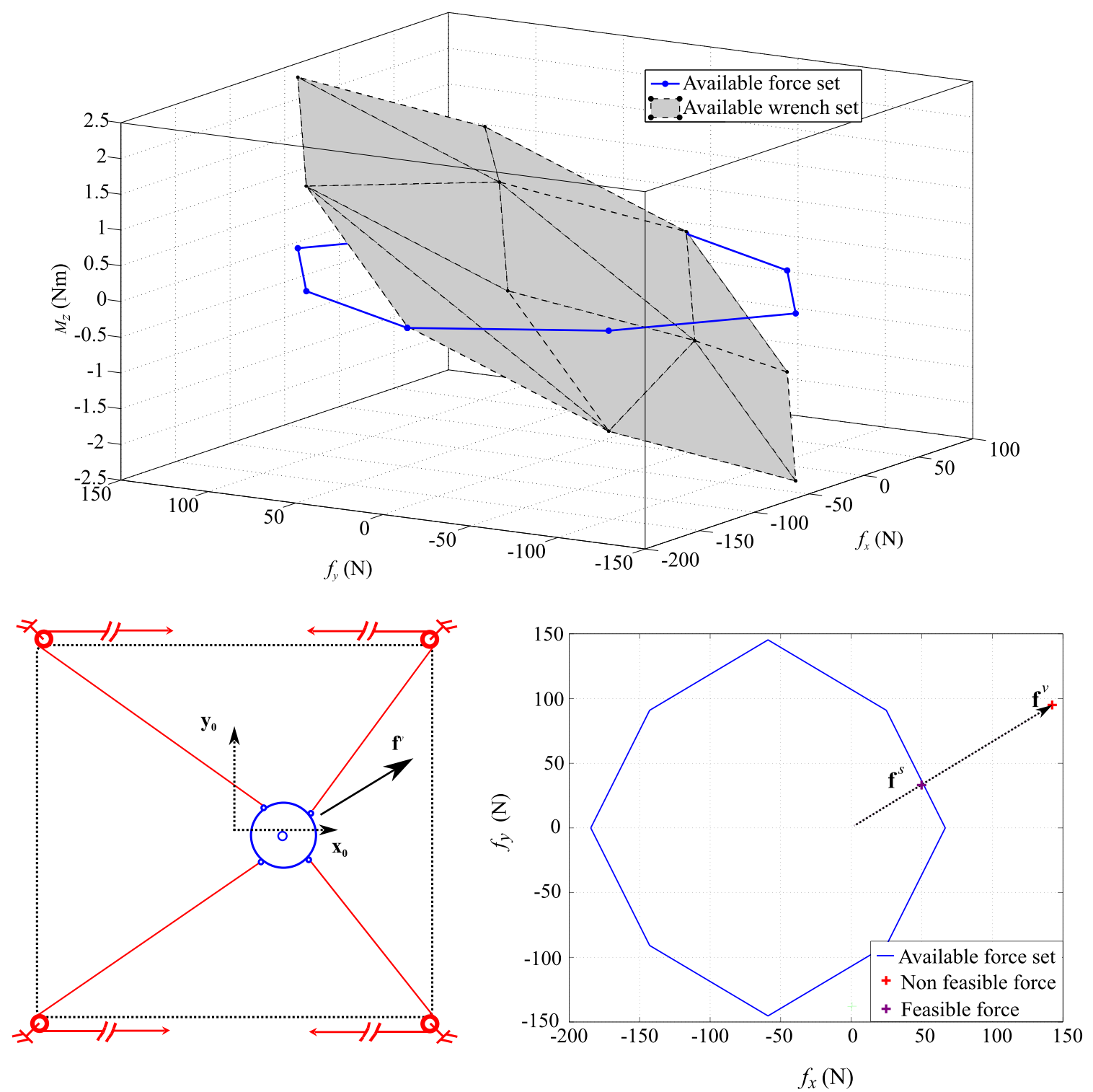

Fig. 5. Boundaries of the AWS (top) defined for the platform position (bottom left). Boundaries of the AFS (bottom right) without considering the moment around $\mathbf{z}_{0}$-axis.

consists in minimizing a weighted infinity-norm of the joint velocity vector that yields to low joint velocities. The approach may however encounter discontinuity issues. Another approach based on the compensation of the saturated joint velocities and/or accelerations is proposed by Omercen et al. [26]. An improved version of this approach has been recently proposed by Flacco et al. [27]. It consists in downscaling the desired cartesian velocity, a virtual force $\mathbf{f}^{v}$ in our case, if this latter is not feasible. The downscaling is performed using an optimal scaling factor so as to satisfy the joint position, joint velocity, and joint acceleration bounds. The approach requires however to compute iteratively the pseudo-inverse of the Jacobian matrix. The feasibility assessment approach we propose avoids these computations. It consists in computing a valid cartesian task, here a cartesian force, that the mechanism is able to counterbalance using admissible tensions in the cables. Note that the approach we here introduce can be easily transposed to solve constrained differential kinematic models of robots.

For a planar robot driven by 4 non-crossed cables, the available wrench set is defined as the set of wrenches (forces and moments) that can be applied to the platform by means of admissible tensions in the cables at a given pose of the platform [28]. The shape of the available wrench set in this case is a spatial zonotope, as represented in the top of Fig. 5. Since the motions of the MRGuide platform are controlled along $\mathbf{x}_{0}$-axis and $\mathbf{y}_{0}$-axis, only the force components $f_{x}$ and $f_{y}$ are represented. Fig. 5 bottom right represents this Available Force Set (AFS) that the mechanism is able to generate regardless of the moment values. The obtained octagon is computed by the projection of the spatial zonotope in the plane $\left(f_{x} f_{y}\right)$. In this example, the minimum and the maximum tensions in the cables are set respectively to $10 \mathrm{~N}$ and $60 \mathrm{~N}$. 
Now, to find a solution to Eq. (2), the virtual force $\mathbf{f}^{v}$ must belong to the AFS. If $\mathbf{f}^{v}$ does not belong to AFS, there are no solutions to Eq. (2) whatever the tension distribution algorithm employed. The feasibility approach described in Algorithm 1 is therefore used in combination with the control scheme. This algorithm, inspired from [28], includes in particular a saturation subroutine, named the ForceSaturation subroutine, which computes a saturation force $\mathbf{f}^{s}$ that the cables are able to counterbalance if $\mathbf{f}^{v}$ is not feasible.

Algorithm 1. Feasibility pseudocode.

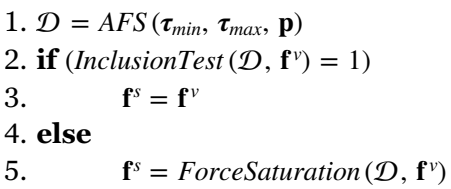

To compute $\mathbf{f}^{s}$, the feasibility pseudocode (cf. Algorithm 1) receives the virtual force $\mathbf{f}^{v}$, the platform position $\mathbf{p}$ and the admissible tensions in the cables $\boldsymbol{\tau}_{\min }$ and $\boldsymbol{\tau}_{\max }$. First, the AFS procedure determines the boundaries $\mathcal{D}$ of the AFS. Then, the InclusionTest procedure verifies if $\mathbf{f}^{v} \in \mathcal{D}$. If the inclusion is satisfied, InclusionTest returns 1 and $\mathbf{f}^{s}=\mathbf{f}^{v}$. Otherwise, InclusionTest returns 0 and the ForceSaturation subroutine, based on the dichotomy process, is launched (cf. Algorithm 2).

Algorithm 2. ForceSaturation subroutine.

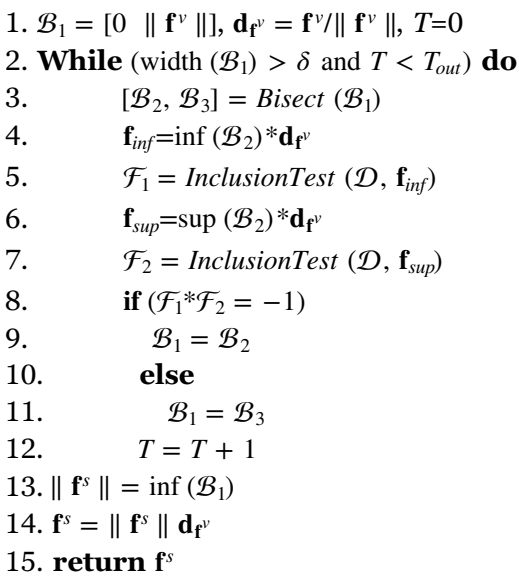

In step 1 of Algorithm 2, the direction $\mathbf{d}_{\mathbf{f}^{v}}$ of the virtual force $\mathbf{f}^{v}$ and the interval $\mathcal{B}_{1}$ in which the bisection process is performed are defined. The lower and upper bounds of $\mathcal{B}_{1}$ are respectively set to 0 and $\left\|\mathbf{f}^{v}\right\|$. The bisection process is stopped if the width of $\mathcal{B}_{1}$ is inferior to a defined threshold $\delta$ or a defined timeout $T_{\text {out }}$ is exceeded. In step 3, the bisection of $\mathcal{B}_{1}$ produces two intervals $\mathcal{B}_{2}$ and $\mathcal{B}_{3}$. In steps 4 and 6 , the forces $\mathbf{f}_{\text {inf }}$ and $\mathbf{f}_{\text {sup }}$ are computed respectively for the lower and upper bounds of $\mathcal{B}_{2}$. The inclusion of $\mathbf{f}_{\text {inf }}$ and $\mathbf{f}_{\text {sup }}$ in $\mathcal{D}$ is tested in steps 5 and 7. If $\mathbf{f}_{\text {inf }} \in \mathcal{D}$ and $\mathbf{f}_{\text {sup }} \notin \mathcal{D}$, this means that the intersection point between the straight line supported by the vector $\mathbf{f}^{v}$ and $\mathcal{D}$ belongs to the interval $\mathcal{B}_{2}$. Thus, the interval $\mathcal{B}_{2}$ is retained (steps 8 and 9). Otherwise, the interval $\mathcal{B}_{3}$ is kept (step 11). The lower bound of $\mathcal{B}_{1}$ is finally used to define the saturation force $\mathbf{f}^{s}$ that is in the same direction as $\mathbf{f}^{v}$ (steps 13 and 14 ).

\subsubsection{Cables pretension}

Since the tensions in the cables are null or near-zero at rest, cables pretension must be performed. This step allows us to avoid cables tensions discontinuity once the positioning control begins. Moreover, if a simple pretension procedure is considered without taking into account the control of the platform position, platform displacement may occur as friction along the cables transmission is not equivalent. To handle the two issues, we propose to control the platform position during the pretension using the same control scheme of Fig. 4. To do so, the minimum and maximum admissible tensions in the cables, $\boldsymbol{\tau}_{\min }$ and $\boldsymbol{\tau}_{\max }$, are increased linearly at each sampling period during the pretension. The desired tensions in the cables $\boldsymbol{\tau}^{d}$ are then computed accordingly by solving Eq. (2). Thus, the platform displacements are handled by the control scheme and the pretension procedure is achieved by modifying the admissible tensions in the cables in the tension distribution block. At the end of the pretension, $\boldsymbol{\tau}_{\min }$ and $\boldsymbol{\tau}_{\max }$ are respectively 10 and $60 \mathrm{~N}$. These values are maintained during the trajectory tracking. In this way, the cables tension discontinuity is avoided between the two phases.

\section{Evaluation and results}

To evaluate this control strategy with the proposed feasibility and pretension algorithms, the set-up of Fig. 2 is considered. The MRGuide prototype is positioned in an horizontal plane and is connected to the actuators using 350-mm long cables. The control 


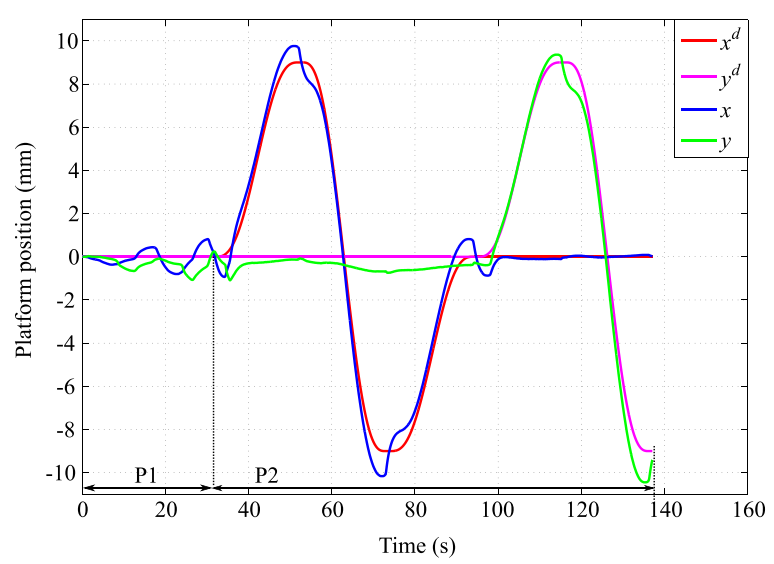

Fig. 6. Trajectory tracking using the control scheme of Fig. 4.

scheme is implemented in $\mathrm{C}$ in a real-time machine, with $1 \mathrm{kHz}$ sampling frequency. Both the desired cables tension computed by the tension distribution algorithm and the measured tensions are represented in Fig. 7. The minimum and maximum admissible tensions $\boldsymbol{\tau}_{\min }$ and $\boldsymbol{\tau}_{\max }$ are set respectively to $10 \mathrm{~N}$ and $60 \mathrm{~N}$.

During the pretension (Fig. 7, phase P1), the gains of the tension controller $c_{\tau}=k_{p \tau}+k_{i \tau} / s$, with $s$ the Laplace variable, are adjusted empirically. The platform desired position $\mathbf{p}^{d}$ is set to $\left[\begin{array}{ll}0 & 0\end{array}\right]^{T}$. Initially, the proportional gains $k_{p x}$ and $k_{p y}$ of the position controller $\mathbf{C}_{\mathbf{p}}$ are considered identical, equal to $k_{p x}=k_{p y}=5.10^{3} \mathrm{Nm}^{-1}$ while the integral gain $k_{i \tau}$ of the tension controller is set to zero. The proportional gain $k_{p \tau}$ of the tension controller is gradually increased until the tension loop becomes unstable. Then in a second
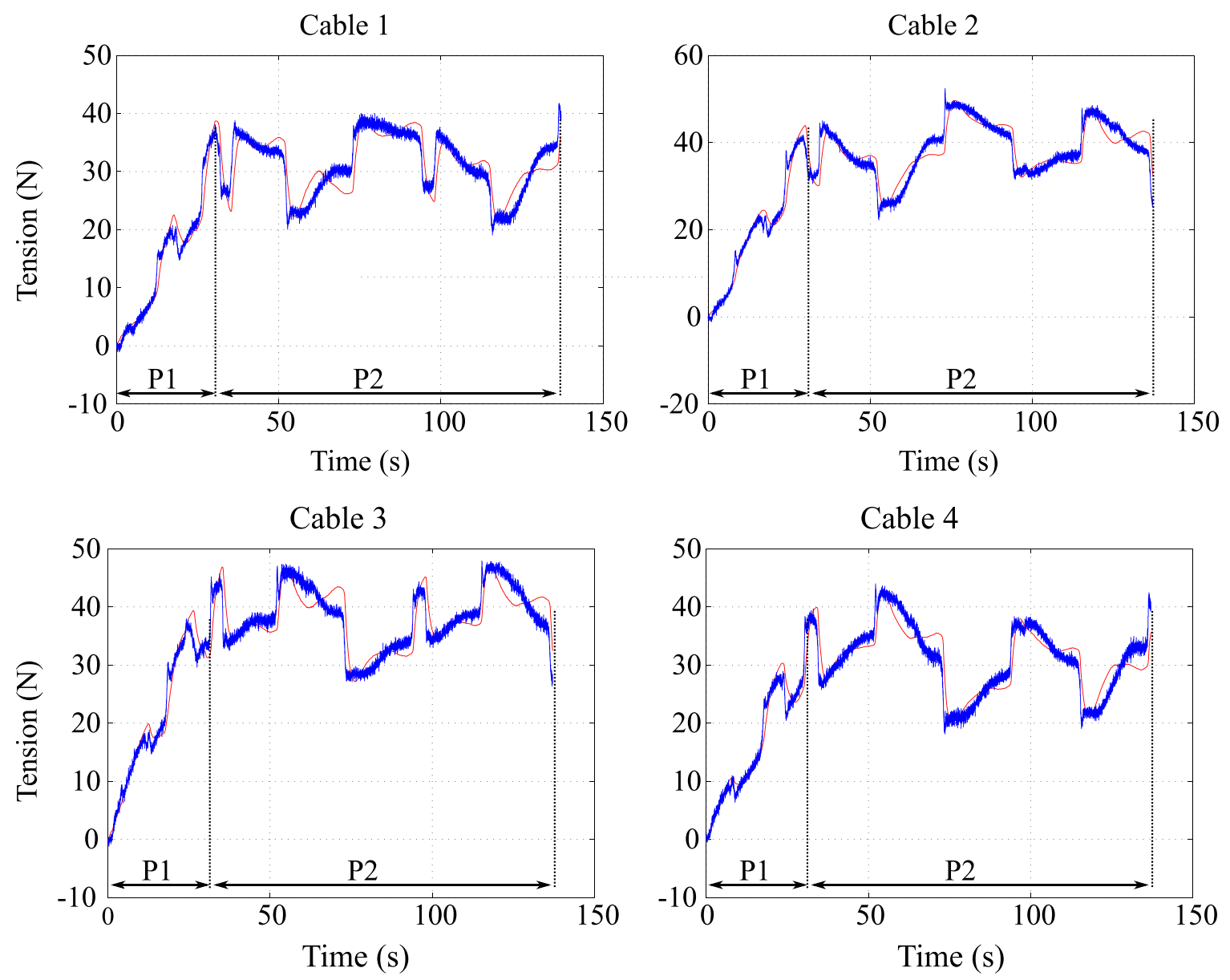

Fig. 7. The desired (red) and the measured (blue) tensions in the cables obtained using the control scheme of Fig. 4. (For interpretation of the references to color in this figure caption, the reader is referred to the web version of this paper.) 


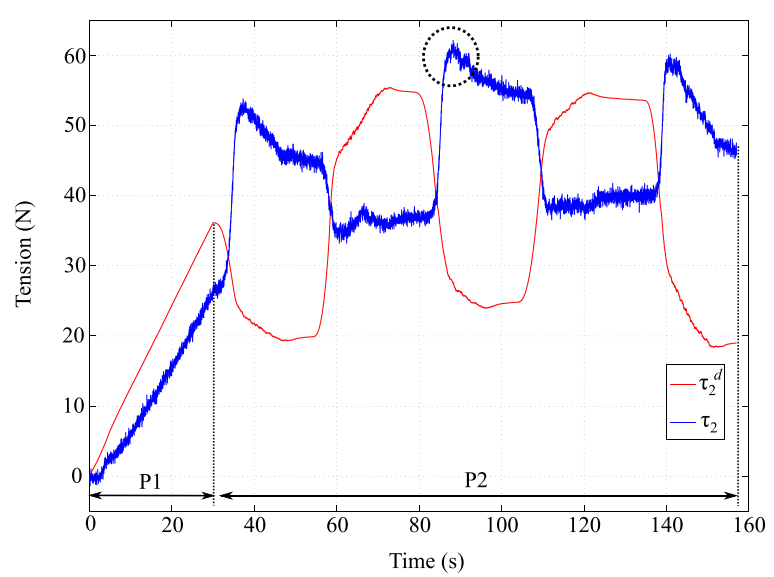

Fig. 8. Desired (red) and measured (blue) tensions for cable 2 without cables tension inner loop. (For interpretation of the references to color in this figure caption, the reader is referred to the web version of this paper.)

Table 1

Comparison between the two control strategies.

\begin{tabular}{lll}
\hline RMS errors & Position control & Position control with tension supervision \\
\hline $\mathbf{x}_{0}$-axis $(\mathrm{mm})$ & 0.7 & 0.7 \\
$\mathbf{y}_{0}$-axis $(\mathrm{mm})$ & 0.5 & 0.4 \\
Cable 1 $(\mathrm{N})$ & 26.8 & 3.4 \\
Cable 2 $(\mathrm{N})$ & 31.1 & 3.3 \\
Cable 3 $(\mathrm{N})$ & 18.1 & 3.5 \\
Cable 4 $(\mathrm{N})$ & 18.3 & 3.2 \\
Mean energy $(\mathrm{mJ})$ & 185 & 56 \\
\hline
\end{tabular}

step, the gain $k_{p \tau}$ is substantially decreased whereas the gain $k_{i \tau}$ is increased gradually. With this empirical adjustment, the best result is obtained with $k_{i \tau}=0.15 \mathrm{rad} \mathrm{N}^{-1} \mathrm{~s}^{-2}$ and $k_{p \tau}=0.5 \mathrm{rad}\left(\mathrm{N} \mathrm{s}^{-1}\right.$.

The pretension procedure lasts $30 \mathrm{~s}$ before switching to trajectory tracking (Fig. 6, P2). As it can be observed, the desired tensions are continuous during the two phases P1 and P2, which validates the pretension procedure. Besides, the tensions in the cables are not equivalent. This is due to the fact that friction along the cables between the structure and the actuators are different. This tension difference in the cables results in less than $1 \mathrm{~mm}$ displacement of the platform (Fig. 6, P1).

Position tracking is assessed by performing a displacement along $\mathbf{x}_{0}$-axis in both directions, and then along $\mathbf{y}_{0}$-axis (Fig. 6, P2). The gains of the position/tension controllers used for the pretension procedure are maintained during the position tracking. As it can be observed in Fig. 6, the tracking performance are satisfactory, with only 10\% overshoot. This latter is acceptable for the application as it represents less than $1 \mathrm{~mm}$. Besides, a very good tension tracking is observed in Fig. 7 .

To evaluate the importance of controlling the cable tension, the same control structure of Fig. 4 is considered but without the internal control loop. This strategy is proposed by Galina et al. [11] for conventional CDM. Identical pretension and position tracking are therefore performed. As the tension tracking is similar for the 4 cables, we have chosen to plot only the tension evolution of cable 2. As shown in Fig. 8, a big difference between the desired tension $\tau_{2}^{d}$ and its measurement $\tau_{2}$ is observed. This difference comes from the fact that friction along the cable transmission is not handled. Moreover, the tension in cable 2 goes beyond the maximum admissible tension of $60 \mathrm{~N}$ in the time range [80, 100] s of Fig. 8. If higher position gains were applied to improve the position tracking, more important tension overshoots would occur without being able to evaluate them given the measurement range of the tension sensors. These results show clearly the interest of the inner tension control loop in the control scheme.

Now to compare quantitatively the two control schemes, the position and tension tracking RMS errors are computed. As it can be noticed from Table 1, the position RMS errors are nearly equivalent for the two schemes. The tension RMS errors, computed for the 4 cables, are however much lower for the proposed control strategy with an average improvement of 85\% in the tension tracking errors. Besides, the average energy consumed by the system is $70 \%$ lower for the proposed control strategy (Table 1). Considering the tension loop in the control scheme has allowed us to reject properly friction between the structure and the actuators. Consequently, the control strategy we propose provides satisfactory results in terms of position tracking and largely improves the tension tracking.

\section{Conclusion}

$\mathrm{CDM}$ represent interesting solutions to deal with strong design constraints as encountered for instance in medical robotics. It is in this context that we have developed a remote actuation cable-driven robot. It is a non-conventional CDM, as the actuators are not 
placed within the manipulator base frame but at a distance using cable transmission for MRI compatibility. High frictions along the line transmission are therefore unavoidable given the manipulator size and the employed materials. In this context, as modeling and compensation of friction are out of reach, a dedicated cascade control strategy has been proposed.

In this paper, this control strategy has been detailed and implemented. To avoid damage during operation using this control strategy, the feasibility and the pretension procedures have been introduced and implemented. As demonstrated, it is possible to control the platform position while supervising the tensions in the cables using the cascade control structure. The advantages of this strategy over a simple position-based control structure have been experimentally demonstrated. Generally speaking, this research study has shown that it is possible to control finely a remote actuation device by a proper combination of instrumentation and control. Such an approach opens the door to new designs in different contexts where compactness is an issue.

\section{References}

[1] K. Chinzei, N. Hata, F. Jolesz, R. Kikinis, Mr compatible surgical assist robot: system integration and preliminary feasibility study, Med. Image Comput. Comput. Assist. Interv. (2000) 921-930. http://dx.doi.org/10.1007/978-3-540-40899-4_95.

[2] A. Goldenberg, J. Trachtenberg, W. Kucharczyk, Y. Yi, M. Haider, L. Ma, R. Weersink, C. Raoufi, Robotic system for closed-bore MRI-guided prostatic interventions, IEEE/ASME Trans. Mechatron. 13 (3) (2008) 374-379. http://dx.doi.org/10.1109/TMECH.2008.924122.

[3] H. Su, M. Zervas, G. Cole, C. Furlong, G. Fischer, Real-time mri-guided needle placement robot with integrated fiber optic force sensing, in: Proceedings of the IEEE International Conference on Robotics and Automation, 2011, pp. 1583-1588, http://dx.doi.org/10.1109/ICRA.2011.5979539.

[4] G. Fischer, I. Iordachita, C. Csoma, J. Tokuda, S. DiMaio, C. Tempany, N. Hata, G. Fichtinger, MRI-compatible pneumatic robot for transperineal prostate needle placement, IEEE/ASME Trans. Mechatron. 13 (3) (2008) 295-305. http://dx.doi.org/10.1109/TMECH.2008.924044.

[5] A. Patriciu, D. Petrisor, M. Muntener, D. Mazilu, M. Schar, D. Stoianovici, Automatic brachytherapy seed placement under MRI guidance, IEEE Trans. Biomed. Eng. 54 (8) (2007) 1499-1506. http://dx.doi.org/10.1109/TBME.2007.900816.

[6] S. Abdelaziz, L. Esteveny, P. Renaud, B. Bayle, L. Barbé, M. De Mathelin, A. Gangi, Design considerations for a novel MRI compatible manipulator for prostate cryoablation, Int. J. Comput. Assist. Radiol. Surg. 6 (6) (2011) 811-819. http://dx.doi.org/10.1007/s11548-011-0558-4.

[7] S. Abdelaziz, L. Esteveny, L. Barbé, P. Renaud, B. Bayle, M. de Mathelin, Design of an MRI-compatible cable-driven manipulator with new instrumentation and synthesis methods, ASME J. Mech. Des. 9 (136) (2014) 1-10. http://dx.doi.org/10.1115/1.4027783.

[8] S. Kawamura, H. Kino, N. Cho, High-speed manipulation by using parallel wire-driven robots, Robotica 18 (1) (2000) 13-21. http://dx.doi.org/10.1017/ S0263574799002477.

[9] S. Fang, D. Franitza, M. Torlo, F. Bekes, M. Hiller, Motion control of a tendon-based parallel manipulator using optimal tension distribution, IEEE/ASME Trans. Mechatron. 9 (3) (2004) 561-568. http://dx.doi.org/10.1109/TMECH.2004.835336.

[10] M. Gouttefarde, J. Lamaury, C. Reichert, T. Bruckmann, A versatile tension distribution algorithm for $n$-dof parallel robots driven by $n+2$ cables, IEEE Trans. Robot. 31 (6) (2015) 1444-1457. http://dx.doi.org/10.1109/TRO.2015.2495005.

[11] P. Gallina, A. Rossi, R. Williams, Planar cable-direct-driven robots, Part ii: Dynamics and control, in: Proceedings of the ASME IDETC/CIE Mechanics and Robotics Conference, 2001.

[12] H. Kino, C. Cheah, S. Yabe, S. Kawamura, S. Arimoto, A motion control scheme in task oriented coordinates and its robustness for parallel wire driven systems', in: International Conference on Advanced Robotics, 1999, pp. 545-550.

[13] H. Kino, T. Yahiro, F. Takemura, T. Morizono, Robust PD control using adaptive compensation for completely restrained parallel-wire driven robots: translational systems using the minimum number of wires under zero-gravity condition, IEEE Trans. Robot. 23 (4) (2007) 803-812. http://dx.doi.org/ 10.1109/TRO.2007.900633.

[14] E. Laroche, R. Chellal, L. Cuvillon, J. Gangloff, A preliminary study for H-infinity control of parallel cable-driven manipulators, in: Cable-Driven Parallel Robots, Springer, Berlin, Heidelberg, 2013, pp. 353-369. http://dx.doi.org/10.1007/978-3-642-31988-4_22.

[15] C. Reichert, K. Müller, T. Bruckmann, Robust internal force-based impedance control for cable-driven parallel robots, in: Cable-Driven Parallel Robots, Springer International Publishing, 2015, pp. 131-143. http://dx.doi.org/10.1007/978-3-319-09489-2 10.

[16] A. Salimi, A. Ramezanifar, J. Mohammadpour, K.M. Grigoriadis, N.V. Tsekos, Design and qualification of a parallel robotic platform to assist with beating-heart intracardiac interventions, Mech. Robot. 6 (2) (2014) 021004. http://dx.doi.org/10.1115/1.4026334 (8 pages).

[17] S. Abdelaziz, L. Esteveny, L. Barbé, P. Renaud, B. Bayle, M. de Mathelin, Development of a MR-compatible cable-driven manipulator: design and technological issues, in: Proceedings of the IEEE International Conference on Robotics and Automation, 2012, pp. 1488-1494. http://dx.doi.org/10.1109/ICRA.2012. 6225302.

[18] M.A. Khosravi, H.D. Taghirad, Robust PID control of fully-constrained cable driven parallel robots, Mechatronics 24 (2) (2014) 87-97. http://dx.doi.org/ 10.1016/j.mechatronics.2013.12.001.

[19] A. Ming, T. Higuchi, Study on multiple degree-of-freedom positioning mechanism using wires part i: concept, design and control, Int. J. Jpn. Soc. Precis. Eng. 28 (2) (1994) 131-138.

[20] C.B. Pham, G. Yang, S.H. Yeo, Dynamic analysis of cable-driven parallel mechanisms, in: Proceedings of the IEEE/ASME International Conference on Advanced Intelligent Mechatronics, 2005, pp. 612-617. http://dx.doi.org/10.1109/AIM.2005.1511050.

[21] T. Bruckmann, A. Pott, M. Hiller, Calculating force distributions for redundantly actuated tendon-based stewart platforms, in: Advances in Robot Kinematics, Springer, Netherlands, 2006, pp. 403-412. http://dx.doi.org/10.1007/978-1-4020-4941-5_44.

[22] R. Verhoeven, Analysis of the workspace of tendon-based stewart platforms (Ph.D. thesis)

[23] A. Pott, T. Bruckmann, L. Mikelsons, Closed-form force distribution for parallel wire robots, in: Computational Kinematics, Springer, Berlin, Heidelberg, 2009, pp. 25-34. http://dx.doi.org/10.1007/978-3-642-01947-0_4.

[24] L. Mikelsons, T. Bruckmann, M. Hiller, D. Schramm, A real-time capable force calculation algorithm for redundant tendon-based parallel manipulators, in: Proceedings of the IEEE International Conference on Robotics and Automation, 2008, pp. 3869-3874. http://dx.doi.org/10.1109/ROBOT.2008.4543805.

[25] A. Deo, I. Walker, Minimum effort inverse kinematics for redundant manipulators, IEEE Trans. Robot. Autom. 13 (5) (1997) 767-775. http://dx.doi.org/ $10.1109 / 70.631238$.

[26] D. Omrcen, L. Zlajpah, B. Nemec, Compensation of velocity and/or acceleration joint saturation applied to redundant manipulator, Robot. Auton. Syst. 55 (4) (2007) 337-344. http://dx.doi.org/10.1016/j.robot.2006.10.001.

[27] F. Flacco, A. De Luca, O. Khatib, Motion control of redundant robots under joint constraints: saturation in the null space, in: 2012 IEEE International Conference on Robotics and Automation (ICRA), 2012, pp. 285-292.

[28] S. Bouchard, C. Gosselin, B. Moore, On the ability of a cable-driven robot to generate a prescribed set of wrenches, ASME J. Mech. Robot. 2 (1) (2010) 1-10. http://dx.doi.org/10.1115/1.4000558. 